
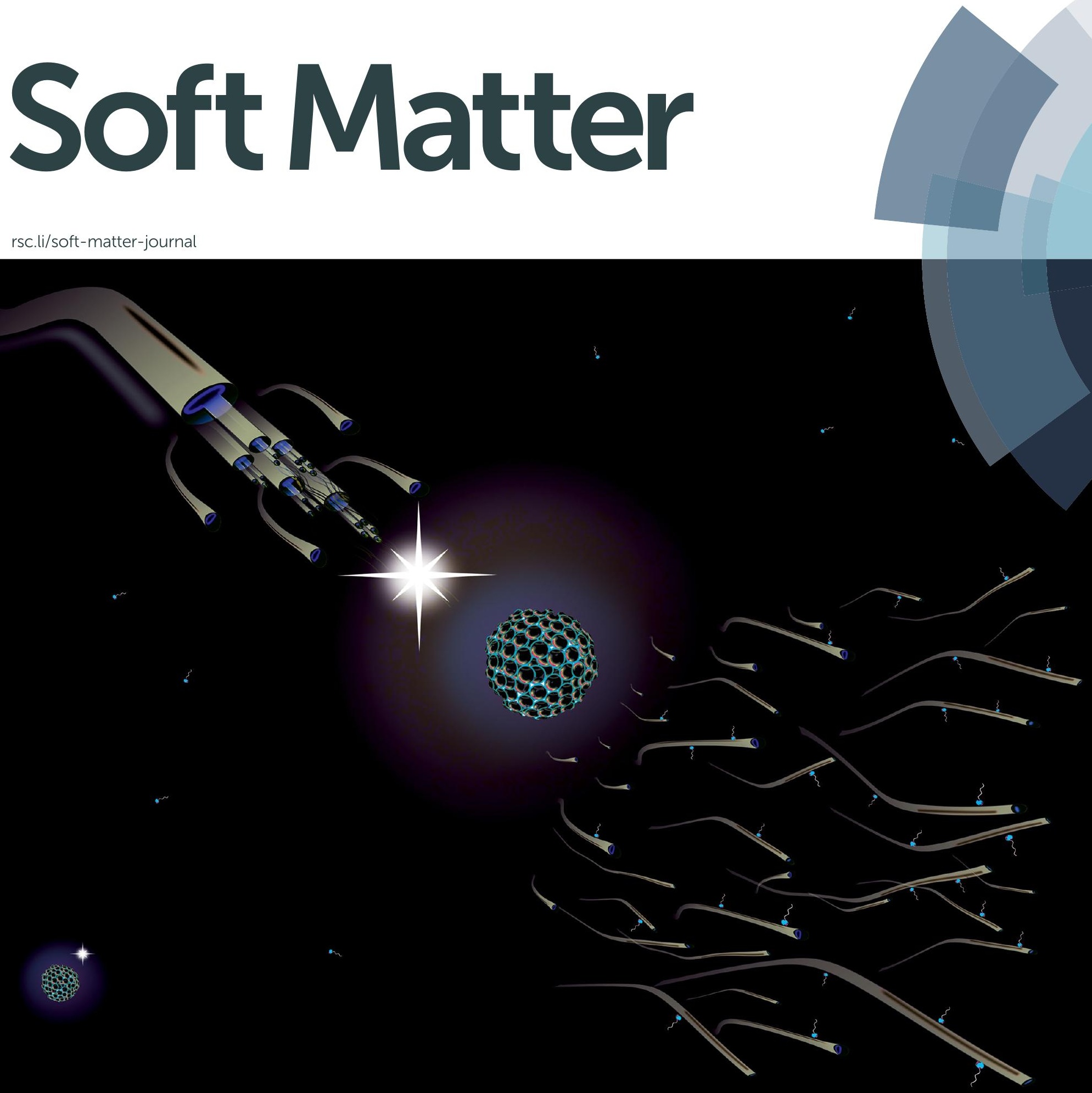
Check for updates

Cite this: Soft Matter, 2018, 14,7793

Received 11th April 2018, Accepted 30th July 2018 DOI: $10.1039 / \mathrm{c} 8 \mathrm{sm} 00752 \mathrm{~g}$ rsc.li/soft-matter-journal

\section{Surfactant controlled zwitterionic cellulose nanofibril dispersions $\dagger$}

\author{
Vincenzo Calabrese, ${ }^{a}$ Marcelo A. da Silva, (D) a Julien Schmitt, (D) ${ }^{\text {a }}$

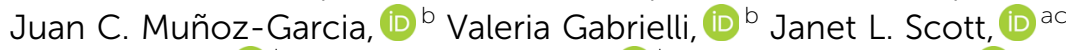 \\ Jesus Angulo, (D) ${ }^{b}$ Yaroslav Z. Khimyak (D) ${ }^{b}$ and Karen J. Edler (D) *a
}

\section{Introduction}

Zwitterionic polymers are a class of polyampholytes, containing anionic and cationic groups with a neutral net charge at a specific $\mathrm{pH} .{ }^{1}$ In the last decade, this class of compounds has received attention for metal binding, ${ }^{2}$ antifouling ${ }^{3-5}$ and antimicrobial $^{6}$ properties and their application in drug delivery ${ }^{7}$ and encapsulation. ${ }^{8,9}$

The self-assembly and the stabilization of zwitterionic moieties depend on $\mathrm{pH}$, ionic strength and temperature $;{ }^{10}$ ideal features for the development of responsive materials. ${ }^{2,11}$ At the isoelectric point (IP), polyampholyte polymers (e.g. proteins) show charge symmetry between the negatively and positively charged moieties leading to flocculation, whereas electrostatic stabilization dominates at $\mathrm{pH}$ values far from the IP. ${ }^{10,11}$ Ionic strength has also been shown to change the conformational arrangement of block polyampholytes and to strongly influence protein solubility, due to a charge screening effect. ${ }^{12,13}$ Although electrostatic stabilization of zwitterionic components is easy to achieve, a drawback is the decreased charge accessibility; a desirable feature for drug delivery. In order to ensure particle stabilization while retaining their surface and charge accessibility,

\footnotetext{
${ }^{a}$ Department of Chemistry, University of Bath, Claverton Down, Bath, BA2 7AY, UK. E-mail: k.edler@bath.ac.uk; Tel: +44(0)1225 384192

${ }^{b}$ School of Pharmacy, University of East Anglia, Norwich Research Park, Norwich, NR4 7TJ, UK. E-mail: j.angulo@uea.ac.uk, y.khimyak@uea.ac.uk ${ }^{c}$ Centre for Sustainable Chemical Technology, University of Bath, Claverton Down, Bath, BA2 7AY, UK. E-mail: j.l.scott@bath.ac.uk; Tel: +44 (0)1225 386307

$\dagger$ Electronic supplementary information (ESI) available: Material characterisation, conductimetric titration and attenuated total reflectance Fourier transform infrared (ATR-FTIR) spectra. See DOI: 10.1039/c8sm00752g
}

depletion stabilization has been previously employed to stabilize several colloidal particles, including gold ${ }^{14}$ and silica particles. ${ }^{15}$ This mechanism occurs when particles, in the presence of nonadsorbing polymers, gain configurational entropy overcoming the attractive osmotic pressure caused by the depletion layer. ${ }^{16}$ This phenomenon occurs at polymer concentrations beyond the concentration required to induce depletion flocculation due to the overlapping of the polymer, which decreases the attractive osmotic energy. ${ }^{16}$

Zwitterionic components, such as proteins, can compete with food supplies, whereas block polyampholytes are not necessarily biodegradable or derived from renewable sources. Here, we describe a novel route for the production of plantbased zwitterionic cellulose nanofibrils (ZCNFs), based on the reported TEMPO-mediated oxidation ${ }^{17-19}$ and glycidyltrimethylammonium chloride (GTMAC) cationization reactions. ${ }^{20,21}$ This functionalization route involves the selective introduction of negatively charged carboxyl groups ${ }^{17,19,22}$ by oxidation, followed by grafting of quaternary ammonium groups from the GTMACcationization. ${ }^{21}$ Although pathways for the production of cellulose nanofibrils (CNFs) bearing oppositely charged moieties have been reported,${ }^{6,7}$ their self-assembly in aqueous media has not, to the best of our knowledge, been described, although the colloidal properties of oxidised cellulose nanofibrils (OCNFs) $)^{18,23-25}$ and quaternary ammonium salt grafted $\mathrm{CNF}^{21,26,27}$ aqueous dispersions have been characterized. Here, we describe the functionalization and double-charged nature of ZCNFs revealed by ${ }^{13} \mathrm{C}$ nuclear magnetic resonance (NMR) spectroscopy and $\zeta$-potential measurements. The relative stability and structure of dispersions of ampholytic ZCNFs in aqueous media containing the anionic surfactant sodium dodecyl sulfate (SDS), cationic 
surfactant dodecyltrimethylammonium bromide (DTAB) and the non-ionic surfactant TWEEN ${ }^{\circledR} 80$ (TW80) were investigated via $\zeta$-potential, transmission electron microscopy (TEM), rheology and small angle neutron scattering (SANS).

\section{Materials}

\section{Sample preparation}

OCNF, prepared using a TEMPO/NaOCl/NaBr oxidation ${ }^{28}$ of wood pulp followed by high-pressure homogenization, was provided by Croda ${ }^{\mathbb{R}}$ International Plc (Goole, UK) as an $8 \mathrm{wt} \%$ slurry. The material was purified by dialysis to ensure that any salts or other process related impurities were removed. In brief, $20 \mathrm{~g}$ of OCNF was suspended in $100 \mathrm{~mL}$ of deionised (DI) water, dispersed by thorough stirring at room temperature, acidified to $\mathrm{pH} 3$ using $1 \mathrm{M} \mathrm{HCl}$ (aq.) and dialysed (Sigma-Aldrich ${ }^{\circledR}$ cellulose dialysis tubing, molecular weight cut-off $12400 \mathrm{Da}$ ) against DI water, for 3 days with daily replacement of DI water. The dialysed OCNF was processed via mechanical shear (Ultra Turrax, IKA T25 digital, $30 \mathrm{~min}$ at $6500 \mathrm{rpm}$ ) and the $\mathrm{pH}$ adjusted to 7 using $0.1 \mathrm{M} \mathrm{NaOH}$ (aq.). After a second dialysis step, the dispersion was diluted to $c a .2 \mathrm{wt} \%$ (dry-basis) and dispersed by sonication (Ultrasonic Processor FB-505, Fisher, $200 \mathrm{~W} \mathrm{~cm}^{-2}$, equipped with a $1 \mathrm{~cm}$ probe) in a series of $1 \mathrm{~s}$ on $/ 1 \mathrm{~s}$ off pulses for a net time of $5 \mathrm{~min}$ at $60 \%$ amplitude in ca. $45 \mathrm{~mL}$ of dispersion contained in an ice bath. Post-purification, stable OCNF dispersions (degree of oxidation (DO) $25 \%$, relative to the hydroxyl group in $\mathrm{C} 6$ of the anhydroglucose units, measured by conductometric titration, ESI, $\dagger$ Fig. $S 1^{17,21}$ ) were prepared. The OCNF dispersion was freeze-dried and used as the substrate for the glycidyltrimethylammonium chloride $(\geq 90 \%)$ (GTMAC, Sigma-Aldrich ${ }^{\mathbb{R}}$ ) functionalization. NaOH powder (15.7 wt\% relative to OCNF dry weight), ultra-pure DI water $(18 \mathrm{M} \Omega \mathrm{cm}$, weight ratio $\mathrm{OCNF} /$ water $=1 / 23)$ and GTMAC $(20 \mathrm{~mol}$ eq. relative to anhydroglucose units (AGUs)) were added to the freeze-dried OCNF. The mixture was stirred for $15 \mathrm{~h}$, heated in a water bath at $65 \pm 1{ }^{\circ} \mathrm{C}$ for 80 min with constant stirring in a sealed vial, cooled to $25^{\circ} \mathrm{C}$ and the reaction quenched by addition of EtOH. To purify the resultant ZCNF dispersion, the suspension was centrifuged ( $5 \mathrm{~min}$ at $3600 \mathrm{RCF}$ ) and the pellet collected and redispersed in DI water; the process was repeated 10 times. This ZCNF dispersion was treated as the stock from which all others were prepared. ZCNFs were dispersed by sonication (Ultrasonic Processor FB-505, Fisher, $200 \mathrm{~W} \mathrm{~cm}^{-2}$, equipped with a $0.63 \mathrm{~cm}$ probe), using a series of $1 \mathrm{~s}$ on/1 s off pulses for $2 \mathrm{~min}$ at $60 \%$ amplitude in $c a .45 \mathrm{~mL}$ of suspension. The $\mathrm{pH}$ of the ZCNF stock dispersion was 6.8 and not adjusted except where otherwise stated. The concentration (wt\%) of the ZCNF stock dispersion was determined by gravimetric analysis and specific ZCNF concentrations were obtained by dilution of the ZCNF stock dispersion with DI water. The ZCNF dispersions at various $\mathrm{pH}$ values were obtained by addition of $1 \mathrm{M} \mathrm{HCl}$ (aq.) or $1 \mathrm{M}$ $\mathrm{NaOH}$ (aq.) and ZCNF dispersions containing the surfactant were prepared by addition of the previously dissolved surfactant and vortexed thoroughly.

\section{Methods}

Rheological analyses were performed using a stress-controlled rheometer (Discovery HR-3, TA instruments ${ }^{\mathbb{R}}$ ) at $25{ }^{\circ} \mathrm{C}$ equipped with a sandblasted plate-plate geometry $(40 \mathrm{~mm})$. The samples were vortexed thoroughly to ensure homogeneity before loading and pre-sheared for $30 \mathrm{~s}$ at a shear rate of $1 \mathrm{~s}^{-1}$ prior to measurement of shear viscosity ( 25 to $100 \mathrm{~s}^{-1}$ ). The viscosity data are presented as the relative viscosity, $\eta_{\mathrm{r}}$, defined as the apparent viscosity, $\eta_{\text {app }}$, normalized by the viscosity of the continuous phase, $\eta_{\mathrm{cp}}$.

$\zeta$-Potential measurements were conducted using a Malvern Zetasizer Nano ZSP ${ }^{\circledR}$. The samples were diluted to $0.01 \mathrm{wt} \%$ ZCNF by addition of water containing $\mathrm{HCl}$ (aq.) or $\mathrm{NaOH}$ (aq.) to achieve a specific $\mathrm{pH}$, or surfactant solution, placed in a capillary electrode cell and the $\zeta$-potential was determined as an average of 4 measurements of 100 scans each.

The shape and size of the ZCNFs were investigated using TEM (JEOL JEM-2100, at $200 \mathrm{kV}$ operating voltage). One droplet of the sample $(0.01 \mathrm{wt} \%)$ was placed on a hydrophilized copper grid (previously glow discharged) and allowed to rest for $30 \mathrm{~s}$, and the excess was removed using filter paper. A droplet of $2 \%$ uranyl acetate was added on top of the grid-sample substrate and the excess was removed after $30 \mathrm{~s}$.

The surface tension at the air water interface $\left(\gamma_{\mathrm{aw}}\right)$ was measured using the Du Noüy ring method (Sigma 701 instrument, Attension ${ }^{\mathbb{R}}$, Sweden) using a $9.58 \mathrm{~mm}$ platinum ring. ZCNF dispersions at $0.001 \mathrm{wt} \%$ were prepared by dilution with DI water and the $\gamma_{\text {aw }}$ measured in a vessel with a diameter of $66 \mathrm{~mm}$. Afterwards, an aliquot of surfactant solution was added to the dispersion (dropwise to the air-water interface) and the evolution of the $\gamma_{w a}$ in the static solution was monitored for $60 \mathrm{~min}$. It should be noted that the surfactant concentrations (125 $\mu \mathrm{M}$ SDS, $125 \mu \mathrm{M}$ DTAB and $2.5 \mu \mathrm{M}$ TW80) were chosen to be below the critical micelle concentration (CMC) of the surfactants. ${ }^{29,30}$ Control samples were prepared using DI water instead of the ZCNF dispersion.

Small angle neutron scattering (SANS) data were collected on SANS2D and Larmor beamlines (Rutherford Appleton Laboratory, Didcot, UK) using wavelengths between 1.5 and $14 \AA$ to obtain a $q$-range between 0.004 and $0.6 \AA^{-1}$. Freeze-dried ZCNF was dispersed in $\mathrm{D}_{2} \mathrm{O}$ by sonication, as described above, and ZCNF dispersions containing surfactant were prepared by addition of the deuterated surfactants, d-SDS and d-DTAB (in dry form, provided by the ISIS Deuteration Facility) and thorough vortex-mixing. Samples were loaded into $1 \mathrm{~mm}$ thick quartz cells. Background subtraction and normalization of the scattered intensity $I(q)\left(\mathrm{cm}^{-1}\right)$ was conducted using the Mantid software routine and data modelling was conducted using the software Sasview 4.0.1. ${ }^{31}$ In agreement with the particle morphology observed by TEM measurements, ZCNFs were modelled as flexible cylinders with elliptical cross sections and uniform scattering length densities, based on the worm-like chain model of Kratky and Porod with the incorporation of the excluded volume effect as described by Pedersen and Schurtenberger. ${ }^{32}$ The Kuhn length, $b_{\text {Kuhn }}$, (twice the persistence length) and the 


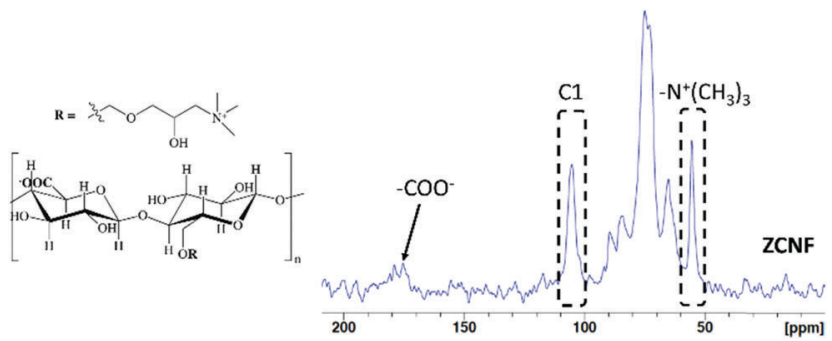

Fig. $1{ }^{13} \mathrm{C}\left\{{ }^{1} \mathrm{H}\right\}$ DP MAS NMR spectra for ZCNF powder (after thorough washing with acid solution ( $\mathrm{pH} 2$ ) and dialysed against $\mathrm{DI}$ water) after 512 scans, acquired on a $300 \mathrm{MHz}$ spectrometer at a $10 \mathrm{kHz}$ MAS rate and an $800 \mathrm{~s}$ relaxation delay. ${ }^{33} \mathrm{~A}$ line broadening of $80 \mathrm{~Hz}$ was used. The peaks corresponding to the anomeric carbon and the cationic group are highlighted within dashed rectangles.

major radius, $r_{\text {maj }}$, of the cross-section were fitted variables while the fibril contour length, $L_{\mathrm{c}}$, and the minor radius, $r_{\min }$, were fixed. The parameter $L_{\mathrm{c}}$ could not be determined within the probed $q$-range, therefore it was fixed at an arbitrary value of $1000 \mathrm{~nm}, L_{\mathrm{c}} \gg\left(2 \pi / q_{\mathrm{min}}\right)$ whereas $r_{\min }$ was fixed to $1 \mathrm{~nm}$, based on the SANS data obtained for $\mathrm{CNF}^{27}$

Solid-state NMR experiments were performed at room temperature using a Bruker Avance III spectrometer equipped with a $4 \mathrm{~mm}$ triple resonance probe operating at frequencies of 300.13 MHz $\left({ }^{1} \mathrm{H}\right)$ and 75.47 $\mathrm{MHz}\left({ }^{13} \mathrm{C}\right)$. Quantitative $1 \mathrm{D}{ }^{13} \mathrm{C}\left\{{ }^{1} \mathrm{H}\right\}$ direct polarization (DP) NMR experiments were conducted at an MAS rate of $10 \mathrm{kHz}$ for ZCNF and OCNF powders tightly packed in an $80 \mu \mathrm{L}$ rotor (Fig. 1 and ESI $\dagger$ Fig. S3). It should be noted that to prove covalent grafting of the GTAMC to the OCNFs, excluding the hypothesis of ionic complexation between the negatively charged carboxyl group and the oppositely charged ammonium group, ${ }^{13} \mathrm{C}$ DP NMR spectra were collected for the freeze-dried powder before and after being thoroughly washed with acid solution ( $\mathrm{pH}$ 2) and dialysed against DI water (Fig. 1 and ESI $\dagger$ Fig. S3). Relaxation delays of 200 (for 4096 scans) and $800 \mathrm{~s}$ (for 512 scans) were employed for the non-washed and washed ZCNF powder, respectively (Fig. 1 and ESI $\dagger$ Fig. S3). It should be noted that an $800 \mathrm{~s}$ recycle delay is sufficient for full relaxation of ${ }^{13} \mathrm{C}$ nuclei in cellulose samples. ${ }^{33}$ All spectra were referenced with respect to TMS. The degree of cationic substitution (DS) of ZCNF was estimated using eqn (1). ${ }^{34}$

$$
\mathrm{DS}=\frac{[\text { area of the methyl carbons resonance at } 55 \mathrm{ppm}]}{[3 \times(\text { area of } \mathrm{C} 1 \text { resonance at } 105 \mathrm{ppm})]}
$$

\section{Results and discussion}

The ampholytic character of ZCNF was revealed by solid-state ${ }^{13} \mathrm{C}$ NMR and $\zeta$-potential measurements. Quantitative solidstate ${ }^{13} \mathrm{C}$ NMR spectra confirmed the presence of the quaternary ammonium group on the ZCNFs (peak at $55 \mathrm{ppm}$ ), ${ }^{34}$ and a DS (eqn (1)) of $23 \%$ was obtained (Fig. 1). In addition, comparison of the $\mathrm{C} 4$ chemical environments in the OCNF and ZCNF ${ }^{13} \mathrm{C}\left\{{ }^{1} \mathrm{H}\right\}$ DP NMR spectra (84 and $89 \mathrm{ppm}$ for the amorphous and crystalline features, respectively ${ }^{35}$ ) showed similar intensity

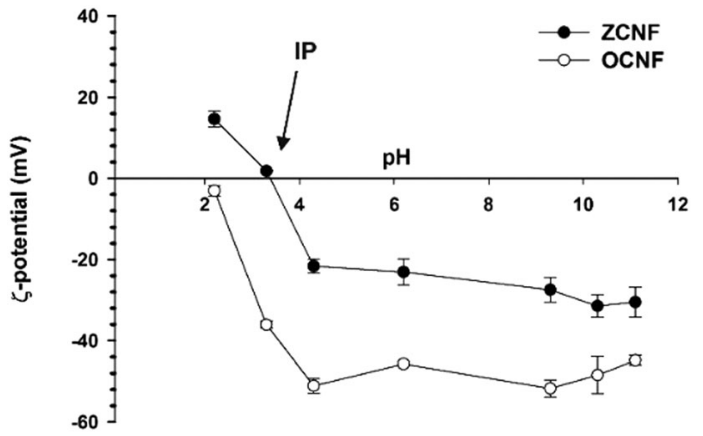

Fig. 2 The $\zeta$-potential of ZCNF and OCNF dispersions as a function of $\mathrm{pH}$. The average and standard deviation of values obtained for duplicate samples are reported.

peaks for the crystalline and amorphous components, indicating that no significant change was caused to the crystalline structure of ZCNF upon GTMAC functionalization (ESI, $\dagger$ Fig. S3). ${ }^{13} \mathrm{C}\left\{{ }^{1} \mathrm{H}\right\}$ DP MAS NMR spectra of both OCNF and ZCNF showed the presence of the carboxyl group carbon atom at $175 \mathrm{ppm}$ (ESI, $\dagger$ Fig. S3). ${ }^{25,36}$ The ${ }^{13} \mathrm{C}\left\{{ }^{1} \mathrm{H}\right\}$ DP MAS NMR spectrum acquired with an $800 \mathrm{~s}$ relaxation delay (Fig. 1), in line with the previously reported estimate for the relaxation delay, ${ }^{33}$ showed the same DS value as that obtained with a $200 \mathrm{~s}$ relaxation delay (ESI, † Fig. S3). +

The $\zeta$-potential of ZCNF was measured across a wide $\mathrm{pH}$ range, from $\mathrm{pH} 2.2$ to 11.1, and further compared with that of the precursor OCNF (Fig. 2). For the $\mathrm{pH}$ range investigated, the $\zeta$-potential of ZCNF is confined between +30 and $-30 \mathrm{mV}$. At $\mathrm{pH}<3.3$, the ZCNF dispersions show a positive $\zeta$-potential, indicating that the positively charged ammonium group outweighs the charge contribution of the protonated carboxyl group. Conversely, at $\mathrm{pH}>3.3$, the net negative $\zeta$-potential indicates that the negatively charged carboxyl group outweighs the positively charged ammonium group. The IP of ZCNF was estimated to be between $\mathrm{pH} 3.3$ and 3.5. As previously reported, the $\zeta$-potential of OCNF remains negative over the range of $\mathrm{pH}$ tested, approaching neutrality at $\mathrm{pH}$ values close to the $\mathrm{p} K_{\mathrm{a}}$ of the carboxyl group. ${ }^{37}$ The presence of oppositely charged moieties on the ZCNF surface strongly affected the stability of ZCNF aqueous dispersions at pH 6.8 upon addition of ionic and non-ionic surfactants. The stability of aqueous ZCNF dispersions was investigated upon addition of negatively charged SDS, positively charged DTAB and non-ionic TW80, referred to henceforth as ZCNF + SDS, ZCNF + DTAB and ZCNF + TW80, respectively. From visual observation, the $0.5 \mathrm{wt} \% \mathrm{ZCNF}$ dispersions in the presence of $25 \mathrm{mM}$ SDS, DTAB and TW80 showed different behaviours (Fig. 3a): $2 \mathrm{~h}$ after preparation, the ZCNF + DTAB dispersion shows similar turbidity to the ZCNF dispersion without surfactant, but ZCNF + SDS exhibits decreased turbidity, suggesting a noteworthy reduction in aggregate size. Although less

$\ddagger$ The DO of $c a$. $25 \%$ was obtained for OCNF by conductometric titration; however, titration was not considered a suitable method to calculate the DO of ZCNF due to the presence of oppositely charged moieties (ESI, $\dagger$ Fig. S1 for conductimetric titration of OCNF and ZCNF). 

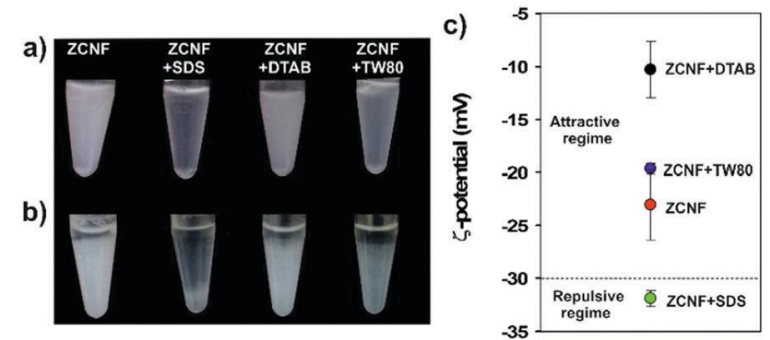

Fig. 3 Images of $0.5 \mathrm{wt} \%$ ZCNF dispersions upon addition of $25 \mathrm{mM} \mathrm{SDS}$, $25 \mathrm{mM}$ DTAB and $25 \mathrm{mM}$ TW80 after (a) 2 hours and (b) 3 days after preparation. (c) The $\zeta$-potential of ZCNF dispersions at $0.01 \mathrm{wt} \%$ upon addition of $25 \mathrm{mM}$ SDS, $25 \mathrm{mM}$ DTAB and $25 \mathrm{mM}$ TW 80 is presented as an average of measurements on duplicate samples.

pronounced than the case of ZCNF + SDS, the ZCNF + TW80 dispersion also shows a decrease in turbidity, suggesting the presence of smaller ZCNF aggregates following TW80 addition. After 3 days, all the dispersions show some sedimentation (Fig. 3b). The ZCNF + DTAB and ZCNF + TW80 samples show similar sedimentation to the surfactant-free dispersion, but the ZCNF + SDS sample shows a smaller quantity of more translucent sediment. Measurement of the particle surface charge has been used to investigate the electrostatic stabilization of $\mathrm{CNF}$ as a function of ionic strength, ${ }^{23} \mathrm{pH},{ }^{37}$ and upon surfactant addition. ${ }^{38}$ As such, the $\zeta$-potential of ZCNF in the presence of $25 \mathrm{mM}$ SDS, DTAB and TW80 was investigated (Fig. 3c).

The pure ZCNF dispersion has a $\zeta$-potential of $-23 \mathrm{mV}$, reflecting the net negative surface charge of the fibrils at $\mathrm{pH}$ 6.8. The ZCNF + SDS dispersion has a greater net negative surface charge compared to the pure ZCNF dispersion $(-32 \mathrm{mV})$, indicating neutralization of the positively charged ZCNF moieties via electrostatic screening provided by the negatively charged SDS. In contrast, the ZCNF + DTAB dispersion shows a decrease in surface charge $(-10 \mathrm{mV})$ due to screening of negatively charged $\mathrm{ZCNF}$ moieties by oppositely charged DTAB. Similarly, Hu et al. reported a decrease in the $\zeta$-potential of negatively charged cellulose nanocrystals upon addition of positively charged surfactants. ${ }^{39}$ It should be noted that for values of $\zeta$-potential between $\pm 30 \mathrm{mV}$, attractive forces are dominant over the repulsive electrostatic forces, leading to flocculation and/or aggregation of the particles. ${ }^{40}$ Thus, the $\zeta$-potential measurements of the ZCNF dispersions suggest electrostatic stabilization upon SDS addition $(\zeta<-30 \mathrm{mV})$ whereas a pronounced instability occurs upon DTAB addition $(-30<\zeta<$ $+30 \mathrm{mV}$ ). Addition of neutral TW80 to the ZCNF dispersion results in an insignificant change in surface charge $(-20 \mathrm{mV})$, as expected for the addition of a non-ionic surfactant, but non-electrostatic driven adsorption of TW80 onto the ZCNF surface could not be excluded by $\zeta$-potential measurements.

In order to probe the non-electrostatic driven adsorption, surface tension measurements were performed. This experiment relies on the fact that the formation of a ZCNF-surfactant complex will exhibit different adsorption kinetics at the air-water interface compared to the individual components.

The $\gamma_{\text {aw }}$ of the ZCNF dispersion did not deviate significantly from that of water $\left(72 \mathrm{mN} \mathrm{m}^{-1}\right.$ at $\left.25{ }^{\circ} \mathrm{C}\right)$, indicating a lack of
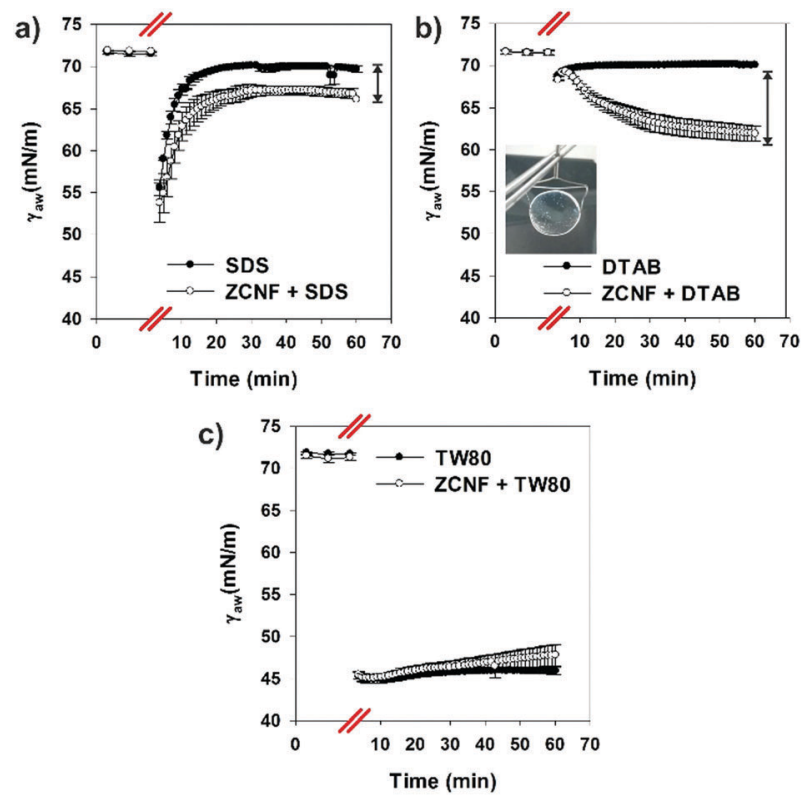

Fig. 4 Surface tension at the air-water interface $\left(\gamma_{\text {aw }}\right)$ of $0.001 \mathrm{wt} \%$ ZCNF dispersions upon addition of (a) $125 \mu \mathrm{M}$ SDS, (b) $125 \mu \mathrm{M}$ DTAB and (c) $2.5 \mu \mathrm{M}$ TW80. The breaks on the $x$ axes indicate the addition of the surfactant to the dispersions. The average of duplicate measurements is reported. The insert in (b) shows the film formed in the Du Noüy ring at the end of the experiment.

surface active properties. Upon addition of SDS (dropwise at the air-water interface) to the ZCNF dispersion, the $\gamma_{\mathrm{aw}}$ decreases dramatically due to the direct addition of the surface active component at the air-water interface, which induces a surfactant and $\gamma_{\text {aw }}$ gradient (Marangoni effect). ${ }^{10}$ Nevertheless, the concentration of surfactant distributed between the air-water interface and the bulk approaches equilibrium with time, reflected in the plateau of $\gamma_{\text {aw }}$ versus time (Fig. 4). The $\gamma_{\text {aw }}$ of ZCNF + SDS is significantly lower than the $\gamma_{\text {aw }}$ of the pure surfactant in water. In agreement with $\zeta$-potential measurements, this phenomenon suggests the formation of ZCNF-SDS complexes, which have greater surface activity compared to the individual ZCNF and SDS. The addition of DTAB to the ZCNF dispersion also leads to a distinct reduction in $\gamma_{\mathrm{aw}}$ compared to pure DTAB in water. The $\gamma_{\text {aw }}$ of the ZCNF + DTAB dispersion does not reach equilibrium within $60 \mathrm{~min}$ and a thin film is slowly formed at the air-water interface (insert image Fig. $4 \mathrm{~b}$ ). This observation supports the hypothesis that the adsorption of the positively charged DTAB onto the negative moieties of ZCNF occurs, neutralising the charge, and rendering the particles more hydrophobic and thus likely to migrate to the air-water interface. Similar phenomena have been previously reported, for example, protein-gum arabic coacervates are more effective at reducing the $\gamma_{\text {aw }}$ than the single components alone.$^{41}$ Moreover, the slow change of $\gamma_{\text {aw }}$ with time, for the ZCNF + DTAB dispersion, points to the presence of slowly migrating aggregates, compared to those occurring in the ZCNF + SDS dispersion, suggesting that larger aggregates are formed in this case. For the ZCNF + TW80 dispersion, the $\gamma_{\text {aw }}$ lies within the standard deviation of the $\gamma_{\text {aw }}$ of the pure surfactant, therefore, the hypothesis 


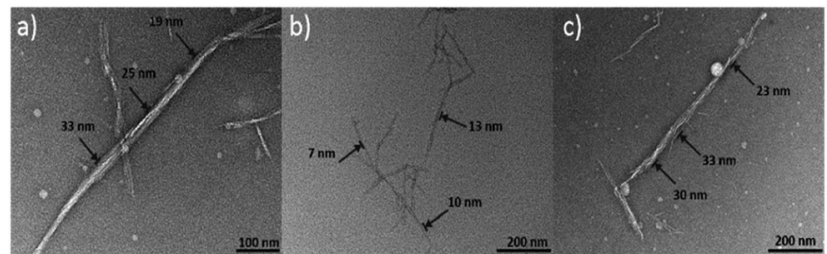

Fig. 5 TEM images of (a) 0.01 wt\% ZCNF dispersion, (b) 0.01 wt\% ZCNF with $50 \mathrm{mM}$ SDS and (c) $0.01 \mathrm{wt} \%$ ZCNF with $50 \mathrm{mM}$ DTAB. Arrows indicate the fibril or fibril aggregate diameters $(\mathrm{nm})$.

of a non-electrostatically driven adsorption of TW80 onto ZCNF can be discarded.

In order to gain information about the shape, size and aggregation of the ZCNFs, TEM images were collected (Fig. 5). The ZCNF dispersion without surfactant shows a pronounced longitudinal fibril-fibril aggregation, resembling thick fibrilbundles with diameters of up to $30 \mathrm{~nm}$, while the ZCNF + SDS dispersion shows individualised fibrils with diameters of ca. $5 \mathrm{~nm}$, similar to the OCNF precursor of ZCNF. ${ }^{25,42,43}$

The presence of individualized fibrils strongly suggests that SDS leads to defibrillation of the ZCNF bundles. The ZCNF + DTAB dispersion shows thick fibril-bundles, as observed for the ZCNF dispersion.

Overall, the TEM images agree with visual observation (Fig. 3) where thick fibril-bundles appear as more turbid dispersions compared to the translucent dispersions with SDS, reflecting individualized and well dispersed fibrils.

In order to gain information about the evolution of the ZCNF aggregates upon surfactant addition, the shear viscosity of $0.5 \mathrm{wt} \%$ ZCNF dispersions was measured at different SDS, DTAB and TW80 concentrations (Fig. 6). A significant reduction in $\eta_{\mathrm{r}}$ occurs even upon addition of $1 \mathrm{mM}$ SDS, a concentration well below the surfactant CMC $\left(7<\mathrm{CMC}<10 \mathrm{mM}^{30}\right)$. A further decrease in $\eta_{\mathrm{r}}$ occurs upon addition of $25 \mathrm{mM}$ SDS and the values of $\eta_{\mathrm{r}}$ remain similar up to $100 \mathrm{mM}$ SDS, indicating that only a minimal quantity of SDS, between 1 and $25 \mathrm{mM}$, is needed to achieve a significant change in rheological properties. In contrast, the addition of different concentrations of DTAB (from 5 to $50 \mathrm{mM}$ ) and TW80 (from 10 to $100 \mathrm{mM}$ ) produces dispersions with similar rheological behaviour to the ZCNF alone dispersions.

The $\eta_{\mathrm{r}}$ of the $0.5 \mathrm{wt} \%$ ZCNF dispersions with $50 \mathrm{mM}$ SDS, DTAB and TW80 were compared based on the severity of the shear thinning behaviour (Fig. 7a). A power law was fitted to the shear viscosity curves and the exponent, corresponding to the slope, was used as an indicator of the shear thinning intensity $\left(\eta_{\mathrm{r}} \sim\right.$ shear rate $^{m}$ ). Upon addition of SDS, the ZCNF dispersion shows a clear transition from shear thinning to an almost shear independent fluid (with a slope change from -0.61 to -0.11 ) alongside a noteworthy decrease in $\eta_{\mathrm{r}}$. These changes in the rheological properties of the ZCNF + SDS dispersion could be assigned to (i) the presence of individualized fibrils and/or thinner fibril-bundles, which easily follow the direction of the flow, supporting the defibrillation suggested by analysis of TEM images (Fig. 5) or (ii) the disruption of aggregates at shear rates below $25 \mathrm{~s}^{-1}$. Conversely, the ZCNF + DTAB dispersion shows a more pronounced $\eta_{\mathrm{r}}$ decay upon

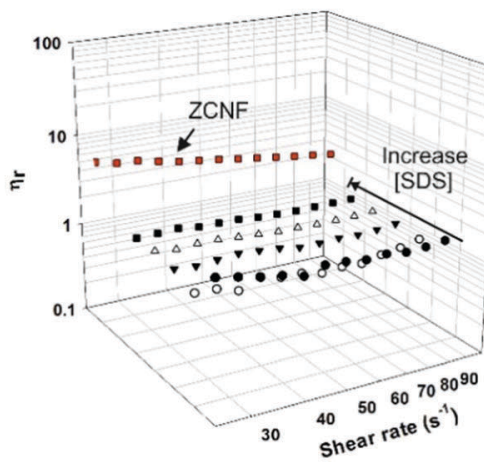

a)

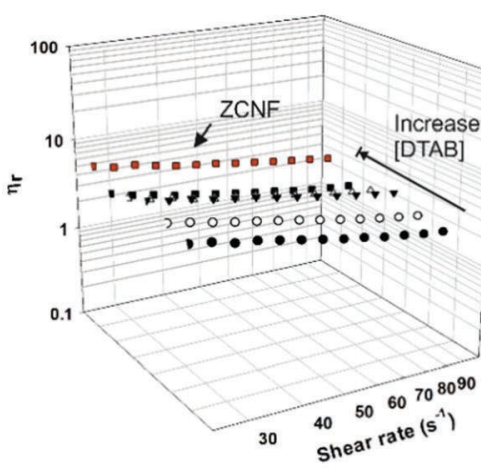

b)

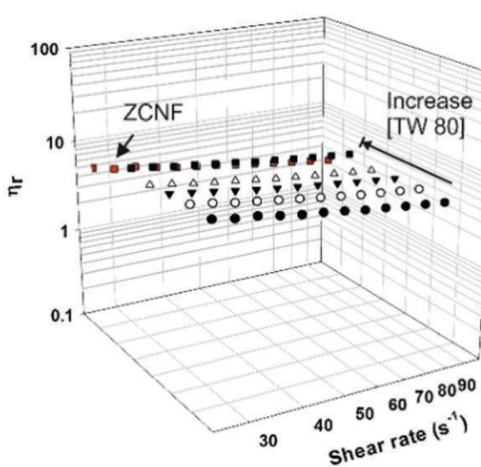

c)

- $\mathrm{ZCNF}+10 \mathrm{mM}$ TW80

- ZCNF $+25 \mathrm{mM}$ TW80

- ZCNF + $50 \mathrm{mM}$ TW80

$\triangle \mathrm{ZCNF}+75 \mathrm{mM}$ TW80

- ZCNF $+100 \mathrm{mM}$ TW80

aCNF

Fig. 6 Relative viscosity $\left(\eta_{r}\right)$ as a function of shear rate $\left(\mathrm{s}^{-1}\right)$ for $0.5 \mathrm{wt} \%$ ZCNF dispersions containing (a) SDS, (b) DTAB and (c) TW80.

application of shear (slope $=-0.71$ ) than the ZCNF dispersion, indicating greater flocculation and the presence of aggregates that are readily disrupted by shear forces. ${ }^{44}$ The ZCNF dispersion containing TW80 shows less pronounced shear thinning behaviour (slope $=-0.50$ ), suggesting less pronounced aggregation. ${ }^{44}$

The weakly shear thinning behaviour of the $0.5 \mathrm{wt} \% \mathrm{ZCNF}$ dispersion containing $50 \mathrm{mM}$ SDS was qualitatively evaluated in terms of flow birefringence and compared to a dispersion of OCNF, the ZCNF precursor, as a positive control. Many studies have shown birefringence in cellulose dispersions and ascribed this phenomenon to the presence of nematic self-ordering of the fibrils. ${ }^{18,26,42,45}$ The $0.5 \mathrm{wt} \% \mathrm{ZCNF}+$ SDS dispersion shows no flow induced birefringence, in contrast to the OCNF dispersion. Therefore, no flow induced alignment of ZCNF occurs in the presence of SDS, while a nematic self-ordering of the fibrils is observed for the OCNF dispersion, as previously reported. ${ }^{45}$

The absence of flow induced self-ordering of the ZCNF + SDS dispersion could be ascribed to the presence of free micelles, 


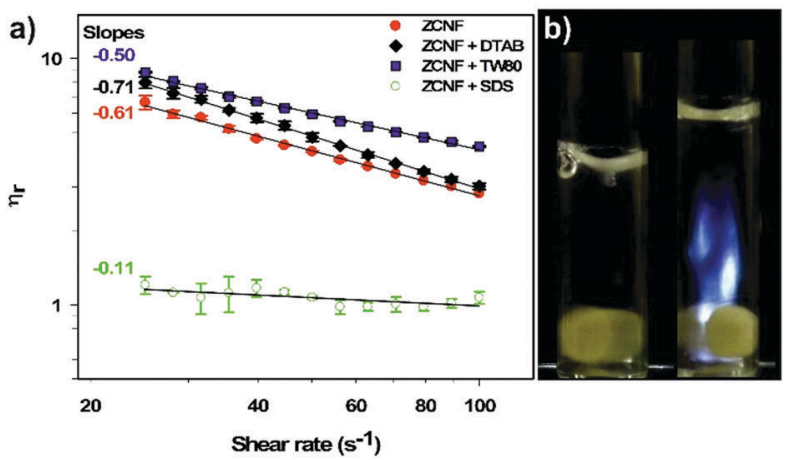

Fig. 7 (a) Relative viscosity $\left(\eta_{r}\right)$ as a function of the shear rate for $0.5 \mathrm{wt} \%$ ZCNF containing $50 \mathrm{mM}$ SDS, $50 \mathrm{mM}$ DTAB and $50 \mathrm{mM}$ TW80. The gradients of the curves (indicated to the left of the curves) are calculated from the values of the exponent of a power law fitting (black line). (b) Flow induced birefringence for a $0.5 \mathrm{wt} \%$ ZCNF dispersion containing $50 \mathrm{mM}$ SDS (left) compared to $0.5 \mathrm{wt} \%$ OCNF (right). The stirred dispersions were photographed between two crossed polarised filters.

leading to steric hindrance, as well as weak hydrophobic interactions between the surfactant tails ionically grafted onto the ZCNF surface. Similar to the ZCNF + SDS dispersion, the pure ZCNF, ZCNF + DTAB and ZCNF + TW80 dispersions exhibited no flow induced birefringence.

SANS analysis provides non-invasive sample measurements, a good statistical overview and the ability to highlight only the ZCNF structure, by contrast matching the surfactant (d-SDS, d-DTAB) to the solvent $\left(\mathrm{D}_{2} \mathrm{O}\right)$. Thus, analysis of SANS data provided the opportunity to compare the aggregation states of ZCNF in the presence of both SDS and DTAB. Data were collected for ZCNF + SDS and ZCNF + DTAB dispersions at surfactant concentrations of 1 and $5 \mathrm{mM}$, respectively. These concentrations were chosen in order to match the lowest surfactant concentrations used in this study. (As d-TW80 is not readily available, contrast matching experiments (surfactantsolvent) with this surfactant were not possible.)

None of the ZCNF dispersions, with or without surfactant, showed the presence of a Guinier region, indicating that the largest dimension of the fibril, the length, is not fully probed within the $q$-range used (Fig. 8). The minimum $q$ probed is $0.005 \AA^{-1}$, thus only features below $125 \mathrm{~nm}\left(2 \pi / q_{\text {min }}\right)$ in size are detected in this experiment. The best data fitting, for all the dispersions, was obtained using the model of flexible cylinders with an elliptical cross section based on the wormlike micelle model. ${ }^{32}$ In this fitting, the parameter $b_{\text {Kuhn }}$ is descriptive of the polymer flexibility; the smaller the $b_{\text {Kuhn }}$, the more flexible the polymer. However, for particles, such as ZCNF, this definition of $b_{\text {Kuhn }}$ is not necessarily the most appropriate. The values of $b_{\text {Kuhn }}$ described from fitting a semi-flexible cylinder model could suggest a convolution of parameters such as mesh size and entanglement length rather than flexibility of the fibrils. The $b_{\text {Kuhn }}$ values of the ZCNF and ZCNF + DTAB dispersions are similar (Table 1), while for the ZCNF + SDS dispersions, $b_{\text {Kuhn }}$ approaches a value similar to $L_{\mathrm{c}}$ (hence fixed at $1000 \mathrm{~nm}$, Table 1), indicating that the fibrils behave like isolated rigid rods, further supporting the non-interacting behaviour suggested from measurements of relative viscosity. The fitting parameter associated with the elliptical

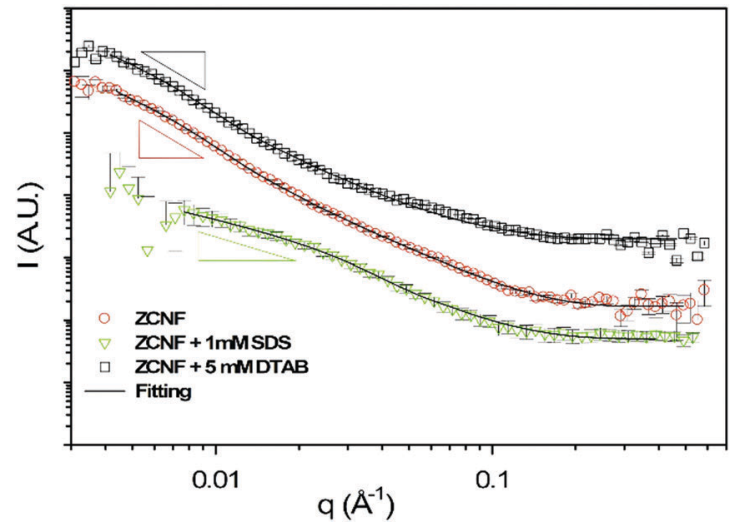

Fig. 8 SANS data for $0.5 \mathrm{wt} \% \mathrm{ZCNF}$ dispersions in the presence of $1 \mathrm{mM}$ $\mathrm{d}$-SDS and $5 \mathrm{mM}$ d-DTAB in $\mathrm{D}_{2} \mathrm{O}$. The black line corresponds to a model of a flexible cylinder with an elliptical cross section used to fit the data. The intensity (I) is showed in arbitrary units (A.U.). Triangles are shown to indicate the power law decay at low-q.

Table 1 Values of the parameters derived from fitting of SANS data for ZCNF and ZCNF plus d-SDS and d-DTAB, 1 and $5 \mathrm{mM}$, respectively, in $\mathrm{D}_{2} \mathrm{O}$. Data were fitted using a model of a flexible cylinder with an elliptical cross section

\begin{tabular}{lllll}
\hline & $r_{\text {maj }}(\mathrm{nm})$ & $b_{\text {Kuhn }}(\mathrm{nm})$ & $\chi^{2}$ & $D_{\mathrm{m}}{ }^{b}$ \\
\hline ZCNF & $27.9 \pm 0.4$ & $19.4 \pm 0.2$ & 2.1 & $2.6 \pm 0.1$ \\
ZCNF + 1 mM SDS & $7.51 \pm 0.1$ & $1000^{a}$ & 2.7 & $1.3 \pm 0.4$ \\
ZCNF + 5 mM DTAB & $27.9 \pm 0.5$ & $23.8 \pm 0.3$ & 5.2 & $2.6 \pm 0.1$
\end{tabular}

${ }^{a} b_{\text {Kuhn }}$ was fixed to the value of $L_{\mathrm{c}} \cdot{ }^{b} D_{\mathrm{m}}$ was calculated in the $q$-range $0.005-0.018 \AA^{-1}$ for ZCNF and ZCNF $+5 \mathrm{mM} \mathrm{DTAB}$, whereas it was 0.008-0.018 $\AA^{-1}$ for ZCNF + $1 \mathrm{mM}$ SDS.

cross section, $r_{\text {maj, }}$, is in agreement with the diameters obtained from measurements of the TEM images (Fig. 5). The power law decay at low- $q$ indicates the fractal-like aggregation of the ZCNFs and the slope indicates the fractal dimension, $D_{\mathrm{m}}$ (Fig. 8). ${ }^{46,47}$ The ZCNF and the ZCNF + DTAB dispersions both yield a value of $D_{\mathrm{m}}=$ $2.6 \pm 0.1$, indicative of dense aggregates. Similar to values derived from small angle X-ray scattering (SAXS) ${ }^{48}$ and SANS ${ }^{49}$ analysis of OCNF dispersions in the absence of surfactant, the ZCNF + SDS dispersion data give $D_{\mathrm{m}}=1.3 \pm 0.4$, indicative of open and less dense aggregates. ${ }^{47}$ From the diffusion limited and reaction limited aggregation models, $D_{\mathrm{m}}$ would be expected to decrease with the increase of the net surface charge, due to the higher number of collisions needed to form the aggregates, until electrostatic stabilization is reached..$^{50}$ Therefore, the smaller value of $D_{\mathrm{m}}$ for ZCNF + SDS suggests that the ZCNF dispersion undergoes a transition from an unstable to a stable regime upon SDS addition. Overall, the difference in $D_{\mathrm{m}}$ between dispersions is in agreement with previous results, where dense ZCNF aggregates show pronounced turbidity, poor stability, and severe shear thinning behaviour compared to the open and less dense aggregates formed upon SDS addition.

\section{Conclusion}

The zwitterionic nature of ZCNF leads to the formation of fibrilbundles that further self-assemble into dense aggregates, 

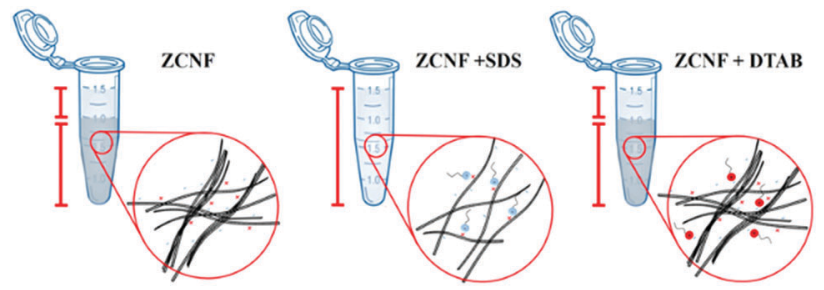

Fig. 9 Schematic of ZCNF dispersions alone and in the presence of negatively charged SDS and positively charged DTAB.

reflected in the fractal dimension $D_{\mathrm{m}}=2.6$, due to attractive electrostatic forces. Addition of negatively charged SDS to the ZCNF dispersion results in electrostatic complexation between SDS and the positively charged moieties present on ZCNF. The ZCNF-SDS complexes lead to electrostatically stabilized dispersions $(\zeta<-30 \mathrm{mV})$, leading to the individualization of fibrils, which form less turbid, more stable, less viscous and weakly shear thinning dispersions (Fig. 9). Upon DTAB addition, the ZCNF negative charge is partially screened due to ZCNFDTAB complex formation leading to greater charge symmetry $(\zeta=-10 \mathrm{mV})$, usually associated with the formation of flocculated aggregates. ${ }^{11}$ The more strongly flocculated nature of the DTAB containing dispersion is evidenced by the greater magnitude of the shear thinning behaviour (slope of the $\eta_{\mathrm{r}}$ curve). Moreover, the similarity in the scattering pattern between the ZCNF and DTAB containing dispersions indicates that the fibril-bundles assemble in a similar fashion. The addition of non-ionic TW80 to ZCNF leads to more translucent and less shear thinning dispersions, as expected for more stable, less flocculated, dispersions. ${ }^{44}$ Therefore, the presence of free TW80 in the dispersion and the enhanced stabilization of ZCNF suggest a mechanism of depletion stabilization induced by the non-adsorbed TW80. Although still a topic of some debate, the depletion stabilization mechanism according to Fleer and Vincent occurs at polymer concentrations beyond the polymer concentration required to induce depletion flocculation and is ascribed to the gain in configurational entropy of the particles over the osmotic attractive energy caused by the depletion layer. ${ }^{16,51}$ Nevertheless, it should be noted that the concentration threshold for a change between the depletion flocculation to depletion stabilization mechanism in ZCNF suspensions was not observed within the concentration range of TW80 investigated, probably due to its low CMC $(12 \mu \mathrm{M}) \cdot{ }^{30}$

\section{Conflicts of interest}

The authors declare no conflicts of interest.

\section{Acknowledgements}

The authors thank the EPSRC for funding this project (grant numbers EP/N033310/1 and EP/N033337/1). V. Calabrese thanks the University of Bath and V. Gabrielli thanks the BBSRC (BB/M011216/1) for PhD studentship funding. The authors would like to thank the ISIS Neutron and Muon Source for the award of beam time (experiment no. RB1710159) and Dr N. Mahmoudi for assistance with the experiment on beamline SANS2D. We acknowledge the ISIS Deuteration Facility for provision of deuterated surfactants. TEM images were obtained at the Microscopy and Analysis Suite in Bath University with the assistance of Ursula Potter. This work benefited from the use of the SasView application, originally developed under the NSF award DMR-0520547. SasView contains code developed with funding from the European Union's Horizon 2020 research and innovation programme under the SINE2020 project, grant agreement No. 654000. Data supporting this article have been made freely available via the University of Bath Research Data Archive system at DOI: 10.15125/BATH-00538 and via the University of East Anglia Data Archive at https://people.uea.ac.uk/en/ datasets/data-for-surfactant-controlled-zwitterionic-cellulosenanofibril-dispersions(3c7b2b6e-42ee-41f5-bb95-97aeece16ce6).html.

\section{References}

1 V. Gold, Compend. Chem. Terminol., IUPAC, 2014, pp. 1622-1622.

2 S. Kudaibergenov and A. Ciferri, Macromol. Rapid Commun., 2007, 28, 1953-1968.

3 A. Venault, W. Y. Huang, S. W. Hsiao, A. Chinnathambi, S. A. Alharbi, H. Chen, J. Zheng and Y. Chang, Langmuir, 2016, 32, 4113-4124.

4 P. Liu, Q. Chen, L. Li, S. Lin and J. Shen, J. Mater. Chem. B, 2014, 2, 7222-7231.

5 R. Yang, H. Jang, R. Stocker and K. K. Gleason, Adv. Mater., 2014, 26, 1711-1718.

6 T. Elschner, C. Lüdecke, D. Kalden, M. Roth, B. Löffler, K. D. Jandt and T. Heinze, Macromol. Biosci., 2016, 16, 522-534.

7 S. Liu, J. Liu, A. R. Esker and K. J. Edgar, Biomacromolecules, 2016, 17, 503-513.

8 P. Trivedi, J. Trygg, T. Saloranta and P. Fardim, Cellulose, 2016, 23, 1751-1761.

9 P. Zhang, F. Sun, C. Tsao, S. Liu, P. Jain, A. Sinclair, H.-C. Hung, T. Bai, K. Wu and S. Jiang, Proc. Natl. Acad. Sci. U. S. A., 2015, 112, 12046-12051.

10 P. Walstra, Physical Chemistry of Foods, CRC Press, 2004, pp. 182-221.

11 M. A. Dyakonova, N. Stavrouli, M. T. Popescu, K. Kyriakos, I. Grillo, M. Philipp, S. Jaksch, C. Tsitsilianis and C. M. Papadakis, Macromolecules, 2014, 47, 7561-7572.

12 M. A. Dyakonova, A. V. Berezkin, K. Kyriakos, S. Gkermpoura, M. T. Popescu, S. K. Filippov, P. Štěpánek, Z. Di, C. Tsitsilianis and C. M. Papadakis, Macromolecules, 2015, 48, 8177-8189.

13 R. M. Kramer, V. R. Shende, N. Motl, C. N. Pace and J. M. Scholtz, Biophys. J., 2012, 102, 1907-1915.

14 X. Zhang, M. R. Servos and J. Liu, J. Am. Chem. Soc., 2012, 134, 9910-9913.

15 S. Kim, K. Hyun, J. Y. Moon, C. Clasen and K. H. Ahn, Langmuir, 2015, 31, 1892-1900.

16 G. J. Fleer, J. H. M. H. Schetjens and B. Vincent, ACS Symposium Series, American Chemical Society, 1984, vol. 240, pp. 245-263. 
17 D. da Silva Perez, S. Montanari and M. R. Vignon, Biomacromolecules, 2003, 4, 1417-1425.

18 T. Saito, T. Uematsu, S. Kimura, T. Enomae and A. Isogai, Soft Matter, 2011, 7, 8804-8809.

19 S. Tsuguyuki and A. Isogai, Biomacromolecules, 2004, 5, 1983-1989.

20 J. C. Courtenay, M. A. Johns, F. Galembeck, C. Deneke, E. M. Lanzoni, C. A. Costa, J. L. Scott and R. I. Sharma, Cellulose, 2016, 24, 253-267.

21 M. Zaman, H. Xiao, F. Chibante and Y. Ni, Carbohydr. Polym., 2012, 89, 163-170.

22 Y. Okita, T. Saito and A. Isogai, Biomacromolecules, 2010, 11, 1696-1700.

23 H. Fukuzumi, R. Tanaka, T. Saito and A. Isogai, Cellulose, 2014, 21, 1553-1559.

24 A. Isogai, T. Saito and H. Fukuzumi, Nanoscale, 2011, 3, 71-85.

25 R. J. Crawford, K. J. Edler, S. Lindhoud, J. L. Scott and G. Unali, Green Chem., 2012, 14, 300-303.

26 M. Hasani, E. D. Cranston, G. Westman and D. G. Gray, Soft Matter, 2008, 4, 2238-2244.

27 J. C. Courtenay, S. M. Ramalhete, W. J. Skuze, R. Soni, Y. Z. Khimyak, K. J. Edler and J. L. Scott, Soft Matter, 2018, 14, 255-263.

28 T. Saito, Y. Nishiyama, J. L. Putaux, M. Vignon and A. Isogai, Biomacromolecules, 2006, 7, 1687-1691.

29 M. A. Bahri, M. Hoebeke, A. Grammenos, L. Delanaye, N. Vandewalle and A. Seret, Colloids Surf., A, 2006, 290, 206-212.

30 D. Linke, Detergents. An Overview, Elsevier Inc., 1st edn, 2009, ch. 34, vol. 463.

31 http://www.sasview.org/contact.html.

32 J. S. Pedersen and P. Schurtenberger, Macromolecules, 1996, 29, 7602-7612.

33 K. Wickholm, P. T. Larsson and T. Iversen, Carbohydr. Res., 1998, 312, 123-129.
34 H. Kono, K. Ogasawara, R. Kusumoto, K. Oshima, H. Hashimoto and Y. Shimizu, Carbohydr. Polym., 2016, 152, 170-180.

35 D. L. VanderHart and R. H. Atalla, Macromolecules, 1984, 17, 1465-1472.

36 C. Tahiri and M. R. Vignon, Cellulose, 2000, 7, 177-188.

37 A. B. Fall, S. B. Lindstrom, O. Sundman, L. Sundman and L. W. Odberg, Langmuir, 2011, 27, 11332-11338.

38 N. Quennouz, S. M. Hashmi, H. S. Choi, J. W. Kim and C. O. Osuji, Soft Matter, 2015, 12, 157-164.

39 Z. Hu, S. Ballinger, R. Pelton and E. D. Cranston, J. Colloid Interface Sci., 2015, 439, 139-148.

40 L. L. Schramm, Advances in chemistry, 1996, vol. 251, pp. 21-22.

41 V. Ducel, J. Richard, Y. Popineau and F. Boury, Biomacromolecules, 2005, 6, 790-796.

42 Y. Habibi, H. Chanzy and M. R. Vignon, Cellulose, 2006, 13, 679-687.

43 T. Saito, S. Kimura, Y. Nishiyama and A. Isogai, Biomacromolecules, 2007, 8, 2485-2491.

44 A. L. Ogden and J. A. Lewis, Langmuir, 1996, 7463, 3413-3424.

45 R. K. Johnson, A. Zink-Sharp and W. G. Glasser, Cellulose, 2011, 18, 1599-1609.

46 F. Cherhal, F. Cousin and I. Capron, Langmuir, 2015, 31, 5596-5602.

47 B. Hammouda, Probing Nanoscale Structures - The SANS toolbox, National Institute of Standards and Technology Center for Neutron Research Gaithersburg, 2010, pp. 211-225.

48 Y. Su, C. Burger, B. S. Hsiao and B. Chu, J. Appl. Crystallogr., 2014, 47, 788-798.

49 Y. Mao, K. Liu, C. Zhan, L. Geng, B. Chu and B. S. Hsiao, J. Phys. Chem. B, 2017, 121, 1340-1351.

50 S. Lazzari, L. Nicoud, B. Jaquet, M. Lattuada and M. Morbidelli, Adv. Colloid Interface Sci., 2016, 235, 1-13.

51 J. Peng, A. Kroes-Nijboer, P. Venema and E. van der Linden, Soft Matter, 2016, 12, 3514. 